\title{
THE GLOBAL INFORMATION SOCIETY AND ELECTRONIC COMMERCE: PRIVACY THREATS AND PRIVACY TECHNOLOGIES
}

\author{
Kathrin Schier $^{1}$ and Simone Fischer-Hübner ${ }^{2}$ \\ 1) EURO Kartensysteme, Frankfurt, Germany \\ 2) Faculty for Informatics, University of Hamburg, Germany
}

\begin{abstract}
In the global information society, privacy is at risk. In this paper, major privacy risks at the communication level and at the application level are analysed. At the application level, we discuss privacy problems of digital payment systems as an example of recently promoted and socially, as well as economically, significant applications for evolving information infrastructures. Moreover, privacy-enhancing technologies are classified and examples of privacy technologies for protecting user identities are given.
\end{abstract}

\section{INTRODUCTION}

Within recent years, most technologically developed countries and the EU have issued information infrastructure programmes for the further development of information highways and to strengthen information and communication industry (e.g. Bangemann 1994). These programmes promote initiatives such as teleworking, distance teaching, health networks and network access to all households with applications such as telebanking. Meanwhile various electronic payment systems have been developed and are partly already in use or tested in pilot projects (Schier 1998a). However, in the global information society, privacy is seriously endangered and is becoming increasingly an international problem. An international harmonisation of privacy legislation in addition to the EU-directive on data protection is therefore needed, but hardly achievable due to cultural differences. Therefore, more privacy enhancing technologies which can technically enforce legal privacy requirements have to be designed, implemented and used.

In this paper, privacy risks in the global information society at the communication level and for the application of digital payment systems are discussed. Furthermore, a short classification and an overview of privacy enhancing technologies are given. 


\section{PRIVACY}

An often used definition of privacy is the one by Alan Westin: "Privacy is the claim of individuals, groups and institutions to determine for themselves, when, how and to what extent information about them is communicated to others" (Westin 1967).

In general, the concept of privacy has three aspects (Rosenberg 1992, Holvast 1993):

- $\quad$ territorial privacy (by protecting the close physical area surrounding a person);

- privacy of the person (by protecting a person against undue interferences, such as physical searches or information violating his moral sense); and

- informational privacy (by controlling whether and how personal data can be gathered, stored, processed or selectively disseminated).

Data protection is the protection of personal data in order to guarantee privacy and is only a part of the concept of privacy.

The emphasis of this paper is on the discussion of the informational privacy of individuals. Individual informational privacy has also been defined by the German Constitutional Court in its Census Decision of 1983 as the term right of informational self-determination, meaning the right of an individual to determine the disclosure and use of his personal data on principle at his discretion.

Privacy Legislation shall protect this fundamental right, if personal data have to be collected or processed. In the EU, privacy protection shall be enforced and harmonised by the EU Directive on the protection of individuals with regard to the processing of personal data and on the free movement of such data (EU Directive 1995). The EU Directive was formally adopted in 1995 by the European Council. It was to be used by member states to amend their respective national laws (where necessary) to comply with the requirements of the directive by 1998 . Besides the privacy protection of individuals, another objective of the EU Directive is to require a uniform minimum standard of privacy protection to prevent restrictions on the free flow of personal data between EU member states for reasons of privacy protection.

The EU Directive on data protection, as well as privacy laws of many western states, requires basic privacy principles to be guaranteed when personal data are collected or processed. These include:

- $\quad$ purpose specification and binding (personal data must be obtained for specified and legitimate purposes and should not be used for other purposes) (see Art. 6 EU Directive);

- necessity of data collection and processing (the collection and processing of personal data shall only be allowed, if it is necessary for 
the tasks falling within the responsibility of the data processing agency) (see Art. 7 EU Directive);

- the data subject's right to information and the right to correction, erasure or blocking of incorrect or illegally stored data (see Art. 10 14 EU Directive);

- control by an independent data protection authority (also called supervisory authority, data protection commissioner, or ombudsman) (see Art. 28 EU-Directive); and

- requirement of adequate technical and organisational security mechanisms to guarantee the confidentiality, integrity, and availability of personal data (see Art. 6,17 EU-Directive).

Privacy enhancing security criteria for the anonymity, pseudonymity or unobservability of a user is derived from the necessity principle. The privacy principle of necessity of data collecting and processing means that personal data should not be collected or used for identification purposes when not really necessary. Consequently, information systems should guarantee that, if possible, users can act anonymously. The best design strategy to enforce this requirement is the avoidance or minimisation of personal data.

\section{THREATS TO PRIVACY IN THE GLOBAL NETWORKED SOCIETY}

In the global information society, privacy is seriously endangered. A key problem is that the traffic on a global network (for example on the Internet) crosses international boundaries, where it can be intercepted or traced, and is not centrally managed. On the Internet, there is no overall responsibility assigned to a certain entity, and there is no international oversight mechanism to enforce legal obligations (especially data protection legislation), as far as they exist (Budapest Draft 1996).

There are severe privacy risks, because personal data about users or other data subjects are available and can be intercepted or traced at different sites around the world.

In the following sections, major privacy risks at the communication level and at the application level will be analysed. At the application level, we discuss privacy problems of digital payment systems as an example of recently promoted and socially as well as economically significant applications for evolving information infrastructures :

\subsection{Privacy threats at the communication level}

A side-effect of global communication is that connection data are available at different sites around the world revealing details about communication partners, time of communication, services used, connections, and so on. These transactional data may reveal who communicated with whom, when, 
for how long, and who bought what for what price. Users leave an electronic trace which can be used to create consumer or communication profiles.

Every electronic message contains a header with information about the sender and recipient, as well as the routing and subject of the message. This information could be intercepted at each site passed. There is normally no anonymity of communication, because the recipient of an electronic mail (even if the email is encrypted) can determine the sender's identity through the sender's email address which normally contains information about the user's name, background (for example, university or company), and location.

If a user is connecting to the Internet by ISDN or telephone, communication data are already obtained by the telephone company. Besides, communication profiles could be created by the service provider to whom the user is connected (like Internet or mailbox providers). Service providers are recording personal user data (such as user name, login name, address, bank connection, and status) as well as accounting data for billing purposes. Users are normally identified and authenticated by the service providers, and their communication behaviour (for example, accesses to news or World Wide Web sites) could be easily traced and supervised by the providers.

Also, personal user data could be recorded at remote servers. A WWW server can record the Internet Protocol (IP) addresses of requesting users, which normally reveals the user's Internet domain, workplace and/or approximate location. Besides, techniques, such as so-called 'cookies' could be used by the remote WWW servers to monitor the user's accesses to web pages. Cookies are blocks of ASCII text that a server can store and later retrieve from the local WWW browser of the user. Cookies, which were introduced by Netscape to allow user-side customisation of Web information, are a mechanism that allows a Web site to record the user's comings and going, usually without his knowledge or consent. If a user is identified by the server as having ordered goods or registered for software, the cookies of this user, revealing his interests in particular web pages, can be related to his name or email address by the server.

Netscape soon modified its browsers so that cookies from one site could not be given to another site. However, web developers and Internet advertising companies (namely Doubleclick Network) soon found a way to use cookies to correlate users' activities between many different web sites. This could be done by adding cookies to GIF images that were served off third-party sites (see Garfinkel et al 1997).

The current cookie usage is violating the provisions of the EU-Directive on data protection and of other national data protection legislation (see also Mayer-Schönberger 1996). First of all, because of their expiration date option, cookies may violate the 'accuracy' and 'timeliness' principles of Art. 6 . Furthermore, the average user is unaware of cookie storage and access. 
However, to meet Art. 7 of the Directive, a user has to give his informed consent to a cookie transfer, since the other alternative conditions of Art. 7 (a legal obligation, vital interests and or contractual arrangements) cannot be assumed. Browsers need to be specifically configured to disallow cookies or to display a cryptic warning that a cookie is going to be stored. The average user has not the technical knowledge to configure his system accordingly or to view or delete the cookie file and he can hardly make an informed decision based on such cryptic warnings. Consequently, there is no informed consent by the user and cookie technology is therefore violating Art. 7 of the Directive. Besides, the extensive information and access rights granted to the user by Art. 10-12 of the Directive is violated.

As pointed out by Goldberg, Wagner and Brewer (1997), privacy threats in the Internet are actually two-fold. The user's online actions could be (1) monitored by unauthorised parties and (2) logged and preserved for access many years later. Long-term databases and powerful search engines make it easy to build a comprehensive profile of individuals and thus contribute to the Internet's 'dossier effect'. For example, "it is technically quite easy to collect information (such as a compendium of all posts you have made to electronic newsgroups) and store it for years or decades, indexed by your name for easy retrieval. If you are looking for a job twenty years from now, do you want your employer to browse through every Usenet posting you have ever made?" (Goldberg et al 1997).

Another problem of the global information society is whether the requirements of appropriate technical and organisational security mechanisms to protect the personal data on the information highways and to provide network reliability can be guaranteed sufficiently. The Internet, an important contemporary information highway that consists of several thousand computer networks with several million users, is known for a lot of critical security holes (Brunnstein et al 1997). The user's privacy is especially endangered by technologies that uses downloaded code, such as JavaScript, Plug-Ins or ActiveX. Downloaded programs can scan the end user's hard disk or network for important information and then smuggle the data to the outside world using the computer's network connection (see Garfinkel et al 1997).

\subsection{Privacy threats of Electronic Payment Systems}

Privacy risks for the global information society are not only existent at the communication level but are also a problem on the application level. To implement an electronic market constituted by electronic transaction, various payment systems are needed. Electronic payment systems are designed to represent ordinary payment systems in an electronic way. As ordinary money, also electronic money can be divided into four categories. There is the system of credit cards, of cheques, of coupons (prepaid tickets) and of money itself. All four types of electronic payment system are already designed or even in use (most of them in pilot projects). A detailed overview 
of different electronic payment systems and their security aspects can be found in (Schier 1997, 1998a).

In the following section, four different electronic payment systems will be described briefly. It will be analysed to what extend they meet the privacy criteria of the anonymity of users and confidentiality of the identities of involved parties and of personal data. The following tables give an overview of how far information can be kept confidential in the different payment systems and how far a user can act anonymously. The entries in the tables show who knows what about whom in the different systems. The columns list the involved partners of a transaction and a possible attacker. The rows list the information that is transmitted for one payment transaction: the identity and the account information of the customer, the identity and the account information of the merchant, the description of the product and the price of the product.

\subsubsection{SET (Digital Credit Cards/ Cheques)}

VISA and MasterCard have jointly developed the Secure Electronic Transaction (SET) Protocol as a method for bank-card transactions over open networks (SET 1996). The SET protocol provides a payment gateway, an institution which organises the money transfer from the customer's bank to the merchant's bank. SET uses cryptography to provide confidentiality of information, payment integrity and authentication of customers and merchants. To use public key cryptography, a certification process is needed producing certificates which are binding the user's identity to the person's public encryption key. A certification hierarchy is needed. The protocol is divided into two phases: the purchase request and the payment authorisation. The purchase request consists of the initiation request and response and a purchase request and response between the customer and the merchant. The second phase consists of the authorisation request and response via the payment gateway.

Table 1. Possible access to personal data in the SET-System

\begin{tabular}{|l|c|c|c|c|c|c|}
\hline SET & $\begin{array}{c}\text { ID } \\
\text { Customer }\end{array}$ & $\begin{array}{c}\text { Account } \\
\text { Customer }\end{array}$ & $\begin{array}{c}\text { ID } \\
\text { Merchant }\end{array}$ & $\begin{array}{c}\text { Account } \\
\text { Merchant }\end{array}$ & $\begin{array}{c}\text { Product } \\
\text { Informa- } \\
\text { tion }\end{array}$ & Price \\
\hline Customer & YES & YES & YES & & YES & YES \\
\hline Merchant & YES & & YES & YES & YES & YES \\
\hline Bank & YES & YES & YES & YES & & YES \\
\hline Attacker & & & YES & & & \\
\hline
\end{tabular}

The SET system protects confidentiality concerning what is known to potential attackers. Attackers do not know the ID of the customer and what 
kind of product is sold, because strong encryption is used. The bank has all information to transfer the money from one party to another except the information about the product itself. The customer is neither anonymous to the merchant, nor to the bank.

\subsubsection{Mondex (Card/Internet based digital Money)}

The concept of digital money proposed by Mondex International (Mondex 1996) provides especially the property of transferability (of money) between private persons. Money, in up to five different currencies, is stored on a chipcard. Therefore, it is more than an Internet payment system. It can also be used in ordinary shops. With an additional device, money can be transferred from one card to another. Both cards have to authenticate each other. A PIN can be used to lock the card, but if the card is 'open' no authentication will happen. The last 10 transactions with detailed information about the customer, the merchant, the product and the price are stored on the card. The merchant stores the last 300 transactions. While transferring money, identification data can be stored in order to prevent unauthorised use. Payment profiles should help to block a card, if it is suspected that the card is misused. According to official statements by Mondex International, this information could be sold for advertising/commercial purpose, but should not.

The Mondex system aimed to be anonymous, but it is not. The bank knows about the transactions, logs them, so that in case of suspicious activities the card can be blocked to prevent unauthorised use. The merchant also stores the last 300 transactions to recognise suspicious use. The last 10 transaction stored in the card can be read with any device without any authorisation from the user. So it could be possible for a merchant, and even for an attacker, to take a look into the log and to create a profile of the customer's activities.

Table 2. Possible access to personal data in the Mondex-System

\begin{tabular}{|l|c|c|c|c|c|c|}
\hline Mondex & $\begin{array}{c}\text { ID } \\
\text { Customer }\end{array}$ & $\begin{array}{c}\text { Account } \\
\text { Customer }\end{array}$ & $\begin{array}{c}\text { ID } \\
\text { Merchant }\end{array}$ & $\begin{array}{c}\text { Account } \\
\text { Merchant }\end{array}$ & $\begin{array}{c}\text { Product } \\
\text { Informa- } \\
\text { tion }\end{array}$ & Price \\
\hline Customer & YES & YES & YES & & YES & YES \\
\hline Merchant & YES & & YES & YES & YES & YES \\
\hline Bank & YES & YES & YES & YES & & YES \\
\hline Attacker & YES & & YES & & & YES \\
\hline
\end{tabular}




\subsection{3 'Geldkarte' (Card based digital Money)}

The electronic wallet is a German add-on to the European eurocheque card. An additional chip is implemented on the magnetic stripe card with the function of an electronic wallet. Money can be loaded on the card (up to DM 400 ) and can be spent in the retail trade. Loading money is only allowed with a special bank terminal which is connected online to the bank authorising system. The amount being loaded is debited from the user's bank account and the loading is logged in a clearing station. The user of the card needs a Personal Identification Number (PIN) to load money on his card, but no authorisation takes place while spending money. The card stores the last 15 transactions. The merchant stores all transactions and sends them to the clearing station at the end of the day. The clearing station collects all transactions on individual clearing accounts and sends the balances of each account to the banks of the involved parties.

The 'Geldkarte' System is not anonymous either, because the customer is known to the merchant and to the bank or clearing station. The bank in cooperation with the clearing station knows everything about a single transaction. The clearing station collects all information in order to handle reclaim cases. In the transaction log, the IDs of the customer and the merchant are coded. Therefore, an attacker could only access the product price information.

\section{Table 3. Possible access to personal data in the Geldkarte-System}

\begin{tabular}{|l|c|c|c|c|c|c|}
\hline Geldkarte & $\begin{array}{c}\text { ID } \\
\text { Customer }\end{array}$ & $\begin{array}{c}\text { Account } \\
\text { Customer }\end{array}$ & $\begin{array}{c}\text { ID } \\
\text { Merchant }\end{array}$ & $\begin{array}{c}\text { Account } \\
\text { Merchant }\end{array}$ & $\begin{array}{c}\text { Product } \\
\text { Informa- } \\
\text { tion }\end{array}$ & Price \\
\hline Customer & YES & YES & YES & & YES & YES \\
\hline Merchant & YES & & YES & YES & YES & YES \\
\hline Bank/Clearing & YES & YES & YES & YES & YES & YES \\
\hline Attacker & & & & & & YES \\
\hline
\end{tabular}

\subsubsection{Ecash (Internet based digital Money)}

Ecash (DigiCash 1996) by the company DigiCash is designed for secure payments from any personal computer to any other workstation, over e-mail or Internet. Ecash is one-sided anonymous. When paying with Ecash the identity of the customer is not revealed automatically. During a payment he can identify himself, but only when he chooses to do so. When clearing a transaction the merchant is identified by the bank. Before Ecash can be used to purchase products it must first be withdrawn from the bank. The withdrawal uses blind signatures (see Chaum 1985, 1992) to prevent the bank from recognising the coins as having come from a particular account. The customer creates the coin himself at random, hides it in a digital 
envelope (by in essence multiplying it with a blinding factor) and sends it off to the bank. The bank withdraws it from the customer's account and makes it 'valid' like an embossed stamp on the envelope (by signing the coin with an unforgeable digital signature indicating its value) before returning it to the customer's computer. The customer removes the blinding factor. Now the money can be spent in a shop or between private persons. When the merchant receives the money, he sends it automatically to the bank and waits for the acceptance before sending the goods to the customer along with a receipt.

Ecash provides only the information that is really necessary for the transaction. The identity of the customer is not published, but the identity of the merchant is visible. The merchant and the bank do not know the identity and the account of the customer, so the customer can perform transactions in an anonymous way as it is possible with ordinary coins. Because of the blind signature system the money cannot be traced back from the merchant or the bank to the customer's account. So the money has the property of anonymity.

Table 4. Possible access to personal data in the Ecash-System

\begin{tabular}{|l|c|c|c|c|c|c|}
\hline Ecash & $\begin{array}{c}\text { ID } \\
\text { Customer }\end{array}$ & $\begin{array}{c}\text { Account } \\
\text { Customer }\end{array}$ & $\begin{array}{c}\text { ID } \\
\text { Merchant }\end{array}$ & $\begin{array}{c}\text { Account } \\
\text { Merchant }\end{array}$ & $\begin{array}{c}\text { Product } \\
\text { Informa- } \\
\text { tion }\end{array}$ & Price \\
\hline Customer & YES & YES & YES & & YES & YES \\
\hline Merchant & & & YES & YES & YES & YES \\
\hline Bank & & & YES & YES & & YES \\
\hline Attacker & & & YES & & & YES \\
\hline
\end{tabular}

Ecash is the only system of the electronic payment schemes introduced above that satisfies the privacy criteria of anonymity. It follows the strategy of avoidance of personal data by using cryptographic mechanisms, especially blind signatures, to provide anonymity for the user. However, in some practical implementation (e.g. the 'Ecash' project of the Deutsche Bank) anonymity is not absolutely enforced, because for each transaction the bank is involved to control the money flow between all parties.

Although Mondex and Geldkarte also use cryptography to enforce confidentiality and integrity, they both do not enable an anonymous use of the systems. SET uses cryptography to protect confidentiality and integrity. In contrast to electronic money systems which aimed to apply to ordinary money like coins and notes, SET was designed for secure credit card transactions over a network which are per se not anonymous.

In most digital payment systems, relevant transactions are logged and audited for security purposes. They can help to detect the unauthorised use 
of systems or even to prevent misuse. The auditing of the logfiles is the weakest point, because the logfiles contain very sensitive information about the financial behaviour of people. These logfiles could e.g. be misused for advertising or marketing purposes. From the privacy viewpoint of course it is very delicate to create profiles about financial behaviour or even to sell them to commercial companies. Thus, a strict regulation of the auditing purpose is needed. The users have to be informed about the usage of the information being collected about them. For that reason (among others) it is necessary to provide anonymous payment systems for the daily use. The electronic form of ordinary payment systems should have at least the same properties as the ordinary ones. Ordinary notes and coins have the property of being anonymous. Thus, the user should have the free choice of using anonymous payment systems whenever he wants, either in an electronic or ordinary way. If non-anonymous payment systems are used it should be known to the user which data is collected and stored. The user has to decide which personal information should be transferred and for what purpose. If different payment systems are combined on one media (for example a smartcard), a security model should be used on the card which ensures the user's informational self-determination. For details about this security model see (Schier 1998b, 1998c).

\subsection{Difficulties of an international harmonisation of privacy legislation}

In conclusion, in the global information society, privacy is at risk and is becoming more and more an international problem. Personal data can easily be transferred or routed across state boundaries to countries without any data protection legislation, where its information content or communication data can be intercepted. The use of most electronic payment systems allows the creation of consumer profiles at the bank or merchant sites which could be situated in different countries. Consequently, internationally harmonised privacy regulations besides the EU Directive are needed for an adequate level of privacy protection.

However, a common harmonised approach to privacy seems to be hardly possible due to cultural, historical, and political differences. Anthropologists have stated that, on a low level, privacy (especially privacy of the person and of the close surroundings) is a human physiological need. But, on higher organisational levels, privacy is basically a cultural construct and there are considerable cultural variations in privacy needs and interests (Lundheim and Sindre 1993). In addition, experiences from World War II, have caused a greater sensitivity to privacy in western European states (Madsen 1992). Another problem can be seen in non-democratic societies, where individual privacy is normally not protected by legislation. On the contrary, in these countries privacy is often invaded by the state.

In (Fischer-Hübner 1997a, 1998) the privacy approaches of technologically developed states that have set up information infrastructure programmes are 
compared with the EU approach. A hope is that the EU Directive will not only be an instrument for harmonisation within Europe. It can also have a coercive effect on countries outside Europe to enact efficient data protection laws based on the EU Directive, because due to Art. 25 of the EU-Directive, there is an economic pressure on non-EU countries to enact efficient data protection acts. According to Art. 25, the export of personal data to third countries (outside the EU), which do not provide an adequate level of data protection, is generally prohibited, subject to some exemptions formulated in Art. 26. For these reasons, East European states have, for instance, already issued new data protection acts according to the EU model and Canada is currently planning to expand its data protection legislation also to the private sector. However, considerable distinctions still exist in the different national approaches to privacy protection. So far, most Asian, African or South American countries do not have any privacy legislation. Besides, there are considerable differences in the privacy approach of the EU and of the USA which has no national privacy law for the private sector and has not established a data protection authority. Thus, in the USA, consumer and credit data can be traded more or less freely. So far, the USA is opposed to enacting data protection legislation according to the European model and instead favours means of self-regulation for the private sector.

The US Department of Commerce has recently proposed 'Safe Harbors' for US companies which would allow US firms to continue exchanging data if the firms choose voluntarily to adhere to certain privacy principles (US Dept. Commerce 1999). The International Safe Harbor Privacy Principles are self-regulatory privacy guidelines that shall prevent US companies' data transfer from being cut off by the EU. However, major problems with selfregulatory approaches are adequacy and enforcement.

\section{PRIVACY ENHANCING TECHNOLOGIES}

In a fully networked society, privacy is seriously endangered and cannot be sufficiently protected by privacy legislation alone. Data protection commissioners are therefore demanding that privacy requirements should also be technically enforced and that privacy should be a design criteria for information systems.

\subsection{Privacy enhancing security aspects}

Security criteria for systems with high privacy requirements should cover a diversity of privacy enhancing security aspects such as:

- Privacy-enhancing security aspects for protecting user identities providing anonymity, pseudonymity, unlinkability, unobservability of users. As discussed above, these criteria are derived from the privacy principle of necessity of data collecting and processing. Anonymity means that a user may use a resource or service without disclosing his 
identity. Pseudonymity means that a user does not disclose his identity, but can still be accountable for his use. Unlinkablity guarantees that a user can make multiple use of a resource or service, but others cannot link these uses together. Unobservability means that others cannot observe that a resource or service is used.

- $\quad$ Privacy-enhancing security aspects for protecting usee identities providing anonymity and pseudonymity of data subjects. If personal data (about data subjects that are not necessarily users at the same time) has to be collected, it should be rendered anonymous or pseudonymous as soon as the purposes for which the data was collected permit this. In some cases, some users still need to access the personal data attributes, while other users only need statistical (anonymous) access. Inference controls for instance can be used to restrict users to statistical accesses only and can prevent those users deducing confidential information about some individual.

- Security mechanisms, such as access control or encryption, that are necessary to protect the confidentiality and integrity of personal data, if (non-anonymous) personal data has to be stored, processed or transmitted. Such security mechanisms can also be classified as data protection technologies. Especially the privacy requirements of purpose binding and necessity of data processing of personal data of users and data subjects can be technically enforced through appropriate security policy and access control mechanisms. (see Fischer-Hübner 1994, 1997b; Fischer-Hübner and Ott 1998) for a formal privacy enforcing access control model).

As discussed in (Rannenberg 1994), none of the early security evaluation criteria, such as the American TCSEC (TCSEC 1985) or the European ITSEC (ITSEC 1991), really covers user and privacy friendly functionality, as their focus is biased on the protection of system owners instead of users and usees. The harmonised Common Criteria (CC 1998) are at least covering the functionality class Privacy for the evaluation of the functionalities Anonymity, Pseudonymity, Unlinkability and Unobservability of users.

\subsection{Classification of privacy enhancing technologies for protecting user identities}

In the global information society, special privacy technologies will be needed that provide anonymity, pseudonymity, unlinkability and/or unobservability for users to prevent the creation of communication and consumer profiles. The Dutch Data Protection Authority (the Registratiekamer) and the Information and Privacy Commissioner (IPC) for the Province of Ontario, Canada, have collaborated in the production of a report (Registratiekamer/IPC 1995) exploring privacy enhancing technologies that safeguard personal privacy by minimising or eliminating 
the collection of identifiable data, and thus permitting transactions to be conducted anonymously. A recent report by a working group of the German data protection commissioners also addresses privacy-enhancing technologies for protecting user identities (Datenschutz-beauftragte 1998). The report and a prior study of the Registratiekamer (Registratiekamer 1995) introduce the concept of an 'identity protector' which is a useful concept to classify transaction systems for which anonymity, pseudonymity, unlinkability and/or unobservability can be provided for the users to certain extent. We therefore also use the concept of an identity protector (IP) to give an overview of important privacy technologies available for protecting user identities which are only partly mentioned in (Registratie-kamer 1995).

As described in (Registratiekamer 1995), an information system is modelled by a user representation (i.e. user process), a service provider representation (which could be a main frame computer or the system of an Internet provider), services (e.g., communication services, payments, access to data), an access control information database (storing the user's privileges) and an audit file (recording all security-relevant actions). The identity protector (IP) can be seen as a system element that controls the exchange of identity between the various system elements. It is installed on the interaction lines in the information system (see Figure 1, next page) and creates two domains within the information system: the identity domain in which the user's identity is known, and the pseudo domain in which the user's identity is secret. In the following sections, different ways of installing identity protectors are discussed:

\section{Placing IP between the services and the other elements of the information system}

Services are located in the pseudo domain and the identity of the user could be kept confidential from other users. However, other system elements remain in the pseudo domain and consequently the user's identity is known by the service provider and is used in the audit file and in the access control information database.

An example for this kind of integrity protector installation, as discussed in (Registratiekamer 1995), is the ISDN caller line identification with the possibility of blocking the transfer of phone numbers. In (Reichenbach et al 1997) a Personal Reachability Management System is introduced for controlling personal reachability in mobile communication while maintaining a high degree of privacy. By easy negotiation of their communication requests users can reach others without disturbing the called partners and without compromising their own privacy. 


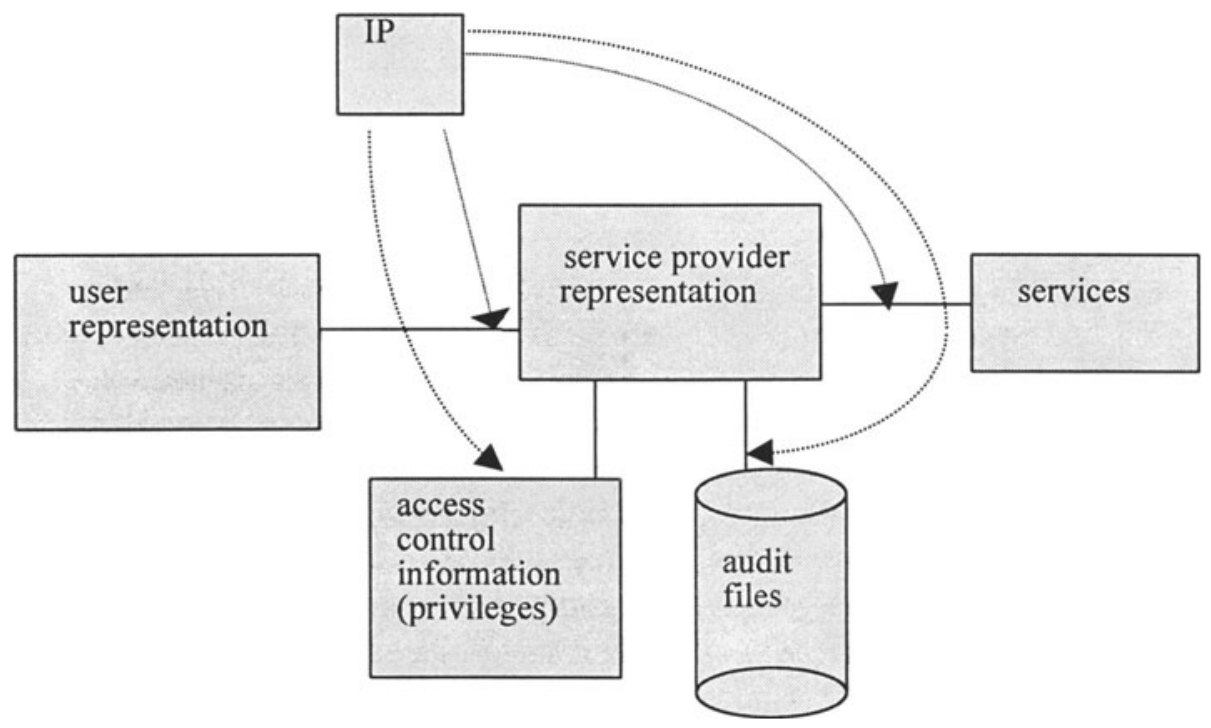

Figure 1. Possible installations of the Identity Protector (IP)

Placing IP between the auditing file and the service provider representation The audit file is contained in the pseudo domain. The service-provider does not register the user's privileges and /or actions under his real identity, but under a pseudo-identity. The concept of pseudonymous auditing which was first presented in (Fischer-Hübner 1993) and later further elaborated and implemented (see Sobirey et al 1997), is an example of this kind of IP installation.

Auditing provides the recording and analysis of security-relevant activities in a system in order to detect and deter intrusions. Thereby it generates personal data about the activities and behaviour of the users which could be misused for other purposes (e.g. employee performance monitoring). Pseudonymous Auditing can balance the conflict between accountability and privacy. It is a privacy enhancing auditing technique, where user identifying data in audit records are pseudonymised after creation and are analysed in pseudonymous representation. When analysing the audit data, the security administrator does not have to know the real user identities. It is sufficient to reidentify a user acting under a pseudonym (ideally with the help of a trusted third party who knows the mappings to real user-IDs), when this user acted suspiciously or obviously intrusive.

Placing IP between the user representation and the service provider representations

In this ideal situation, the identity domain only contains the user representation, i.e. the user identities are unknown to all other system elements. 
Such system concepts, where the identity domain only contains the user representation, can be further classified according to whether they protect the user identities at the communication level, at the system level or at the application level. Examples for such system concepts protecting user identities are:

a) Protection at the communication level

- Sender anonymity by dummy traffic, DC-nets (Chaum 1988) or crowds (Reiter and Rubin 1988);

- $\quad$ Recipient anonymity by message broadcast and implicit addresses;

- Unlinkability of sender and recipient by mix-nets (e.g. anonymous remailers (Cottrell 1995), Onion Routing (Syverson et al 1997), Freedom Network Architecture (Zero-Knowledge Systems 1999)).

DC-nets (Chaum 1988) are based on superposed sending and message broadcast and can provide perfect sender and recipient anonymity against global attackers. However, if there is insufficient bandwidth for the use of the broadcast approach, Mix-nets (Chaum, 1981) seem to be a practically better approach. Mix-nets realise unlinkability of sender and recipient, i.e. sender and recipient cannot be identified as communicating with each other, so that traffic analysis can be prevented. Besides, it enables sender anonymity against the recipient and optionally recipient anonymity.

Anonymous remailers are implementation of mix-technologies for electronic mail and should allow the anonymous use of electronic mail. Simple anonymous remailers are intermediary computers which secretly pass messages to a recipient. However, such remailers cannot sufficiently protect privacy, because a mapping of anonymous identities to real addresses must be maintained by the re-mailer which, for that reason, can be a sensitive point of attack. The Finnish remailer service anon.penet.fi was recently closed down, after it had been raided by the Finnish police in cooperation of the FBI.

So-called cypherpunk remailers enable the chaining of encrypted messages through a series of remailers. The structure of messages is a nested set of encrypted messages, where each message is encrypted to a re-mailer. Cypherpunk remailers should ensure that only the first re-mailer in the chain knows the address of the sender, and only the last re-mailer knows the address of the receiver. However, cypherpunk remailers can be attacked as well (see Cottrell 1995). Messages could be traced, if incoming messages to a re-mailer are directly forwarded. Even if incoming messages are delayed or reordered, an attacker could send a batch of messages after your message arrives, so that your message will be flushed back out of the re-mailer's message pool. Besides, messages could be tracked by their size (which is decreasing) or by the use of active replay attacks.

A re-mailer which can guarantee anonymity effectively is Mixmaster (Cottrell 1995) which is based on the concept of Mixes (Chaum 1981). 
Mixmaster uses constant-length messages, includes defences against replay attacks and offers improved message reordering code to stop passive correlation attacks based on timing coincidence.

Furthermore, there exist implementation of the mix concept for interactive Internet communication, namely Onion Routing (Syverson et al 1997), which was implemented at the Naval Research Laboratory, and the Freedom Network Architecture by Zero-Knowledge Systems (by Zero-Knowledge Systems 1999).

b) Protection at System Level

- Pseudonymous system accounts;

- Anonymous system access through authorisation certificates.

Pseudonymous system accounts, as a simple organisational measure which allows a user to access a system under a pseudonym, were suggested by Registratiekamer (1995). If a new employee enters a large organisation, the head of the department can draw up an access profile for him on the basis of the required access level. This profile, which should not contain data that can be associated with the new employee, is then sent to the system administrator. The system administrator checks the profile for authenticity and sets up a user account under a pseudonymous user-ID with authorisations in accordance with the employee's access profile. He then returns a form to the department head with the user-ID and a password for the new pseudonymous user account. Thus the new employee now has access to the system without the system administrator knowing who the employee is. If the employee is violating his access rights, he can still be identified through the department head.

Besides, authorisation certificates allow the implementation of distributed access control management with the option for a user to anonymously access systems, services and/or resources. Authorisation certificates are digitally signed records that bind a public key to an authority given to the key holder. They usually do not contain identity information about the key holder (in contrast to identity certificates such as X.509 certificates). Thus, they can be used by the keyholder to act anonymously. For example, an authorisation certificate that provides the authorisation to a subject (keyholder) to access a system can be used by this subject to gain anonymous system access, if he can prove that he possesses the corresponding private key by using a public key authentication protocol.

c) Protection at the application level (example: electronic payment systems)

- Anonymous prepaid cards (e.g., telephone cards);

- Anonymous payment systems based on blind signatures (e.g., Ecash, see above). 
Note that the anonymity of an Ecash customers is only protected, if he does not use Ecash over non-anonymised IP connections and if he does not send a delivery address.

\section{OUTLOOK}

On the way to the Global Information Society, privacy enhancing technologies are becoming more and more important. The report of the Registratiekamer and IPC (Registratiekamer/IPC 1995) concludes that, if privacy technologies are to play a more significant role, it will be necessary to create more public awareness as well as consumer demand for them. If there is a demand, providers will probably try to respond to market forces.

However, it is argued that privacy technologies enforcing anonymity for the users could be misused to commit crimes. For example, Mix-nets can be misused to anonymously distribute junk mail, logic bombs or copyrightprotected material, or to hack systems without leaving any traces. A system using blind signatures for guaranteeing anonymous electronic payment can be misused for money laundering or other forms of organised crime (see, for example, Solms and Naccache 1992). Most electronic payment systems are therefore designed to prevent organised crime. For this reason transactions are logged to detect suspicious use.

Nevertheless, outside the electronic world, anonymity is common place and accepted as valuable (e.g., anonymous AIDS lab tests, anonymous telephone hot-lines, anonymous publishing, anonymous cash that allows goods to be bought anonymously). Hence, it should also be preserved in the electronic world.

If there are no real anonymous electronic payment systems, some people will prefer the ordinary form of money because of its anonymity. However, the use of ordinary money could make them suspicious of hiding something or of money laundering. This could endanger the free choice of the different systems for innocent users without criminal intentions. In addition to the risk of being under suspicion when using ordinary money, people who do not use electronic payment systems could also be subject to discrimination. Consequently, also for this reason it is important that the user has the possibility of using anonymous electronic payment systems and generally, of using privacy enhancing technologies.

\section{REFERENCES}

Bangemann, M. (1994) Europe and the global information society: recommendations to the European Council. Brussels, 26 May 1994 (Bangemann report), http://www.earn.net/EC/bangemann.html 
Brunnstein, K. \& Schier, K. (1997) Global digital commerce: impacts and risks for the development of global information societies. Proceedings of the IFIP WG 9.2 Corfu international conference, May 8-10, 1997, Chapman \& Hall, London.

Budapest Draft (1996) International Working Group on Data Protection in Telecommunications, Data Protection on the Internet, Report and Guidance (Budapest Draft), May 1996.

CC (1998) Common Criteria Editorial Board: Common Criteria for Information Technology Security Evaluation, Version 2.0, May 1998.

Chaum, D. (1981) Untraceable electronic mail, return addresses, and digital pseudonyms. Communications of the ACM 24 (2) 84-88.

Chaum, D. (1985) Security without identification: transaction systems to make Big Brother obsolete. Communications of the ACM 28(10) 1030-1044.

Chaum, D. (1988) The dining cryptographers problem: unconditional sender and recipient untraceability. Journal of Cryptology 1 65-75.

Chaum, D. (1992) Achieving electronic privacy. Scientific American, August 1992, pp.76-81.

Cottrell, L. (1995) Mixmaster and re-mailer attacks. http://www.obscura.com/ loki/remailer/re-mailer-essay.html

Datenschutzbeauftragte (1998) Arbeitsgruppe "Datenschutzfreundliche Technologien" der Datenschutzbeauf-tragten des Bundes und der Länder, Arbeitspapier "Datenschutzfreundliche Technologien". http://www.datenschutz-berlin.de/to/datenfr.htm

Digicash (1996) Product Information, Digicash. http://www.digicash.nl

EU (1995) Directive 95/46/EC of the European Parliament and of the Council of 24 October 1995 on the protection of individuals with regard to the processing of personal data and on the free movement of such data.

Fischer-Hübner, S. (1993) IDA (Intrusion Detection and Avoidance System): Ein einbruchsentdeckendes und einbruchsvermeidendes System. Shaker-Verlag, Reihe Informatik, 1993 (in German).

Fischer-Hübner, S. (1994) Towards a privacy-friendly design and use of IT-security mechanisms. Proceedings of the 17th National Computer Security Conference, Baltimore, October 1994.

Fischer-Hübner, S. (1997a) Privacy at risk in the global information society. In Berleur, J. and Whitehouse, D. (eds.) An ethical global information society: culture and democracy revisited. Proceedings of the IFIP WG 9.2 Corfu international conference, May 8-10, 1997, Chapman\&Hall, 1997.

Fischer-Hübner, S. (1997b) A formal task-based privacy model and its implementation: an updated report. Proceedings of the Second Nordic Workshop on Secure Computer Systems NORDSEC'97, Helsiniki, November 6-7, 1997.

Fischer-Hübner, S. (1998) Privacy and security at risk in the global information society. Information, Communication \& Society (iCS) 1(4), Winter 1998, 419-441.

Fischer-Hübner, S. and Ott, A. (1998) From a formal privacy model to its implementation. Proceedings of the 21st National Information Systems Security Conference, Arlington, VA, October 5-8, 1998.

Garfinkel, S. and Spafford, G.(1997) Web security \& commerce. O’Reilly \& Associates, Inc. 
Goldberg, I., Wagner, D. and Brewer, E. (1997) Privacy-enhancing technologies for the Internet. Proceedings of COMPCON'97, San Jose, February, 1997. http://www.cs.berkeley.edu/ daw/privacy-compcon97-www/privacy-html.html

Holvast, J. (1993) Vulnerability and privacy: are we on the way to a risk-free society? In Berleur, J. et al. (eds.) Facing the challenge of risk and vulnerability in an information society. Proceedings of the IFIP-WG9.2 Conference, Namur May 20-22. Elsevier Science Publishers B.V. (North-Holland), 1993.

ITSEC (1991) Information Technology Security Evaluation Criteria (ITSEC), Provisional Harmonised Criteria, June 1991.

Lundheim, R. and Sindre, G. (1993) Privacy and Computing: a Cultural Perspective. In Sizer, R. et al. (eds.): Security and control of Information Technology in Society. IFIP WG 9.6 Working Conference, St.Petersburg, Elsevier Science Publishers.

Madsen, W. (1992) Handbook of personal data protection. Stockton Press.

Mayer-Schönberger, V. (1997) The Internet and privacy legislation: cookies for a threat? West Virginia Journal of Law \& Technology.

Mondex (1996) Product Information, Mondex. http://www.mondex.com/mondex/

$\begin{array}{llllll}\text { Netscape (1997) The Open Profiling } & \text { The }\end{array}$ http://developers.netscape.com/ops/ops.html

Rannenberg, K. (1994) Recent developments in IT security evaluation - the need for evaluation criteria for multilateral security. In Sizer, R. et al (eds.) Security and control of Information Technology in Society. IFIP WG 9.6 Working Conference, St.Petersburg. Elsevier Science Publishers, pp. 113-128.

Registratiekamer (1995) Privacy-enhancing technologies: the path to anonymity, Volume II. Achtergrond-studies en Verkenningen 5B, Rijswijk, August 1995.

Registratiekamer/IPC (1995) Registratiekamer, the Netherlands and Information and Privacy Commissioner/ Ontario, Canada, Privacy-enhancing technologies: the path to anonymity, Volume I, Achtergrondstudies en Verkenningen 5A, August 1995.

Reichenbach, M., Damker, H. and Rannenberg, K. (1997) Individual management of personal reachability in mobile communication. In Yngstroem, L. and Carlsen, J.(eds.) Proceedings of the IFIP TC-11 Sec'97-Conference 'Information Security in Research and Business', Copenhagen, May 14-16. Capman\&Hall, London.

Reiter, M. and Rubin, A. (1988) Crowds: anonymity for web transactions. ACM Transactions on Information and System Security 1(1) 66-92.

Rosenberg, R. (1992) The Social Impact of Computers. Academic Press.

Schier, K. (1997) Vergleich und Bewertung aktueller Systeme im elektronischen Zahlungsverkehr, Proceedings of the German UNIX User Group Conference (GUUG), September 1997 (in German).

Schier, K. (1998a) Zahlungssysteme im Internet - Eine sicherheitstechnische Bewertung, in: Heinen, I. (Hrsg): Internet - von der Idee zum kommerziellen Einsatz, Deutscher Internet Kongreß, dpunkt.verlag, Frankfurt, May 1998.

Schier, K. (1998b) A role and task based security model for multifunctional smartcard applications in the area of electronic commerce. In Papp, G. and Posch, R. (eds.) Global ITSecurity. Proceedings of the IFIP TC-11 SEC 1998 Conference, Wien/Budapest, Kluwer. 
Schier, K. (1998c) Multifunctional smartcard applications for electronic commerce - an application of the role and task based security model. Proceedings of the 15th. Annual Computer Security Application Conference, ACSAC 1998, Phoenix, AR. IEEE Computer Society Press, Los Alamitos.

SET (1996) SET Specification, Visa and Mastercard 1996, http://www.visa.com/cgibin/vee/sf/set/intro.html.

Sobirey, M., Fischer-Hübner, S. and Rannenberg, K. (1997) Pseudonymous auditing for a privacy-enhanced intrusion detection. In Yngstroem, L. and Carlsen, J.(eds.) Proceedings of the IFIP TC-11 Sec'97-Conference 'Information Security in Research and Business', Copenhagen, May 14-16. Chapman \& Hall, London.

Solms, S. v. and Naccache, D. (1992) On blind signatures and perfect crimes. Computers and Security 11 581-583.

Syverson, P., Goldschlag, D. and Reed, M. (1997) Anonymous connections and onion routing. Proceedings of the 1997 Symposium on Security and Privacy, IEEE Computer Society Press, Oakland, 1997, http://www.itd.nrl.navy.mil/ITD/5540/projects/onionrouting/OAKLAND_97.ps, http://www.onion-router.net/Publications.html

TCSEC (1985) DoD Trusted Computer Systems Evaluation Criteria, DoD 5200.28-STD, Washington D.C., Department of Defence, 1985.

US Dept. Commerce (1999) International Safe Harbor Privacy Principles, June DRAFT, 1999.

Westin, A. (1967) Privacy and Freedom. New York.

Zero-Knowledge Systems Inc (1999) White Paper: The freedom network architecture, Version 1.0. http://www.zeroknowledge.com/products/Freedom_Architecture.html 